\title{
Ultrasound-Assisted Lipolysis of the Omentum in Dwarf Pigs
}

\author{
Angela Faga, ${ }^{1}$ Luigi Valdatta, ${ }^{2}$ Marzio Mezzetti, ${ }^{2}$ Mara Buoro, ${ }^{2}$ Alessandro Thione ${ }^{2}$ \\ ${ }^{1}$ Pavia and ${ }^{2}$ Varese, Italy
}

\begin{abstract}
Successful surgical treatment of medium degree obesity by subcutaneous liposuction has been reported in the literature. In obesity, most adipose tissue is visceral, mainly omental, and the resection of omentum is a mutilating procedure for the intestinal tract. Because of this, we planned to reduce omental adipose tissue by an apparently conservative approach: ultrasound-assisted lipolysis.

The purpose of this study was to assess the feasibility and safety of this procedure in an animal experimental model, drawing clinical and autoptic patterns.

We chose pigs because they are functionally analogous to humans, although they store less fat in the omentum, whose structure looks like a veil.

Four male dwarf pigs were fed, since weaning, with hyperlipidic fodder. When they were eight months old, they were operated on under general anesthesia in our laboratory for experimental surgery. After laparotomy, the omentum was delivered and treated with ultrasound for 1 hour. Before and just after the sonication, biopsies were drawn from omentum and processed for histologic findings.
\end{abstract}

After 50 days, the surviving animals were sacrificed and autopsied; specimens from omentum, liver, and spleen were histologically processed.

Two animals died during the operation, while the two surviving animals were in good general condition.

Macro and microscopic observations demonstrated that the ultrasound can liquefy omental fat, sparing its fibrous network in the immediate time; during the postoperative period, an intense inflammatory reaction developed; macroscopic observation evidenced fibrous adhesions of the omentum to the surrounding organs; the connective tissue network was thickened and the whole omentum was twisted on itself.

The high mortality rate could be due either to the surgical learning curve or to casualty or to lethal effects of ultrasound

Correspondence to A. Faga, c/o Plastic Surgery Unit, I.R. C.C.S. Fondazione Salvatore Maugeri, Via Ferrata, 8, 27100 Pavia, Italy; email: afaga@fsm.it on the cardiac conductive system; the inflammatory peritoneal reaction could be specifically due to ultrasound or to surgical handling.

Key words: Ultrasound lipolysis—Omentum—Dwarf pigs

The use of ultrasonic energy for ablative purposes was first described by Fry and Johnson more than 30 years ago [6,7]. From that moment on many different specialities throughout the world have been using ultrasonic energy technique.

In 1988 Zocchi [11] introduced and popularized the use of ultrasound in the treatment of patients undergoing lipoplasty. In 1991 Fournier reported the successful surgical treatment of medium degree obesity by massive aspiration of subcutaneous fat tissue [4,5].

However, in obesity most adipose tissue is visceral, mainly omental; omentum, or epiploon, is a large plica of mobile and well-vascularized peritoneum, which is full of adipose tissue especially in the obese patient. It is placed in front of the abdominal organs. The omentum has been defined the "abdominal policeman" by Morrison [9] because of its important function of protecting the abdominal organs from perforations, infections, ischemic episodes, and so on. It is a structure which is often utilized in many surgical fields $[3,10]$. We thought to remove omental adipose tissue with ultrasound assisted lipolysis because this technique has proven effective in liquefying subcutaneous adipose tissue, sparing the connective and the vascular framework $[4,5]$.

The goal of this study was to investigate the feasibility and safety of this procedure in an animal experimental model, drawing autoptic and histological patterns.

The pig model was chosen because functionally analogous to humans, although pigs store less fat in the omentum. 


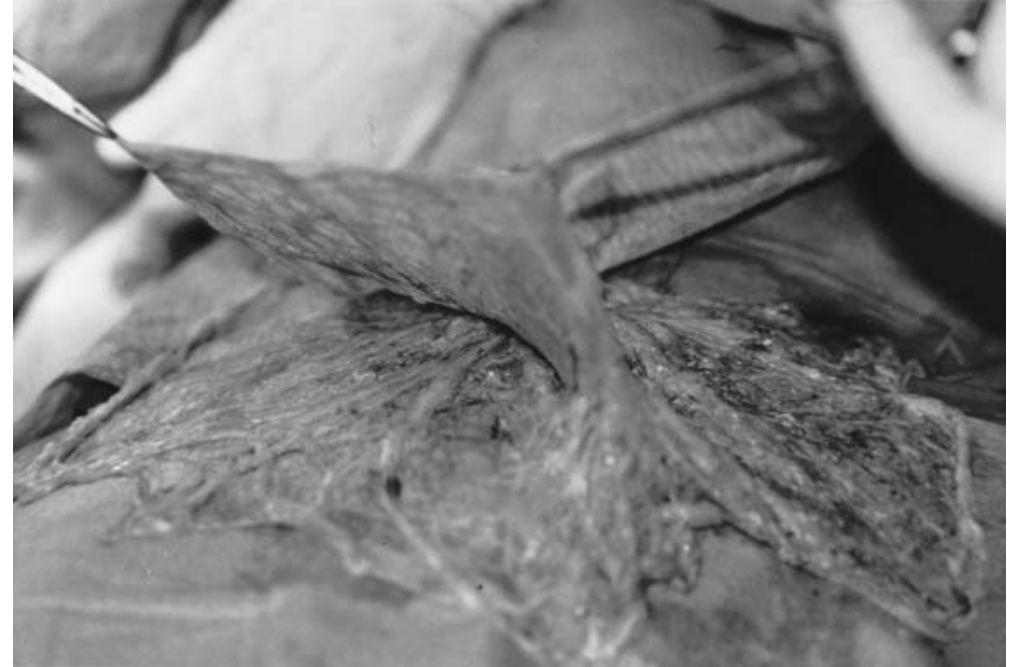

Fig. 1. Intraoperative: the omentum after sonication. Adipose tissue is reduced and the thin connective network is spared.

\section{Materials and Methods}

The experiment was divided in three parts:

- Operative phase

- Autoptic phase

- Histopathologic phase

Four male dwarf pigs, weighing approximately $35 \mathrm{~kg}$, were used for this experiment. Each animal was caged according to the rules stated by the European Laws on experimental animals' treatment. From weaning until the day before surgery they were fed with hyperlipidic fodder, in order to increase the deposit of fat both in the subcutaneous and in the intraabdominal compartments. At the age of eight months, they were operated in our laboratory for experimental surgery, which is equipped with everything needed to perform and monitor surgery (Experimental Surgery Laboratory, University of Insubria, Varese, Italy).

The animals were kept fasting for 24 hours before surgery in order to avoid anesthetic complications. Anesthesia was achieved with atropine $(0.04 \mathrm{mg} / \mathrm{kg})$, ketamine $(15 \mathrm{mg} / \mathrm{kg})$, midazolam $(0.5 \mathrm{mg} / \mathrm{kg})$, and intubation by tracheotomy utilizing isoflurane. Electrocardiac and respiratory activity were monitored and an ossimeter for blood saturation was positioned at the ear.

The preoperative health status of each animal was determined by recording oxygen level in arterial blood, heart rate, and respiratory rate. A perioperative intravenous single-dose prophylactic antibiotic was performed (ceftriaxone $2 \mathrm{~g}$ ). The abdomen was shaved with a hairclipper and the operative area was prepared with Iodopovidone solution. After xipho-ombelical laparotomy, the omentum was delivered and laid down on wet gauzes. Biopsies were drawn from the gastric insertion and from the distal margin of the omentum.

Then we started the treating with ultrasounds $(27 \mathrm{kHz}$, $50 \mathrm{~W}$ ) using the prototype system named Mentor Contour Genesis Integrated System ${ }^{\circledR}$, developed by Mentor
Corporation, Santa Barbara, CA. It consists of the following instrumentation: an electrical power console, a handpiece with the piezoelectric transducer, and a $5 \mathrm{~mm}$ titanium cannula with an external sheath which allowed irrigation of the operative field with saline solution during the procedure.

The liquefaction of adipose tissue was completed in about $1 \mathrm{~h}$. The omentum was then washed out with saline and gently settled back into the abdomen.

Immediately after the sonication, biopsies were drawn from the omentum and processed for histology. The laparotomic wound was routinely repaired. After a $24 \mathrm{~h}$ postoperative monitoring, the animals were sent back to the stabling house, where they were fed a normal caloric diet.

During the follow-up period, the animals were in good general conditions.

After 50 days, the surviving animals were sacrificed and autopsied and specimens from omentum, liver, and spleen were drawn and histologically evaluated (Pathology Unit, Ospedale di Circolo, Varese, Italy).

\section{Results}

The dwarf pigs' omentum delivered from the abdominal cavity before surgery looked roseate, with a connective network including vessels and a fair quantity of adipose tissue.

After the operative phase we observed the immediate results of ultrasounds on omental tissues: the apron was intact and apparently the connective and vascular frameworks were perfectly spared without bleeding signs nor local burns (Fig. 1). Two animals died during the operation, after sonication just before waking up from anesthesia. Immediate autopsy gave evidence to death due to cardiac conductive system failure: while suturing the parietal peritoneum, we observed a sinusal tachicardy with heart rate ranging from 130 to 190 pulses per minute, followed by a large complexes arrhythmia with heart rate 

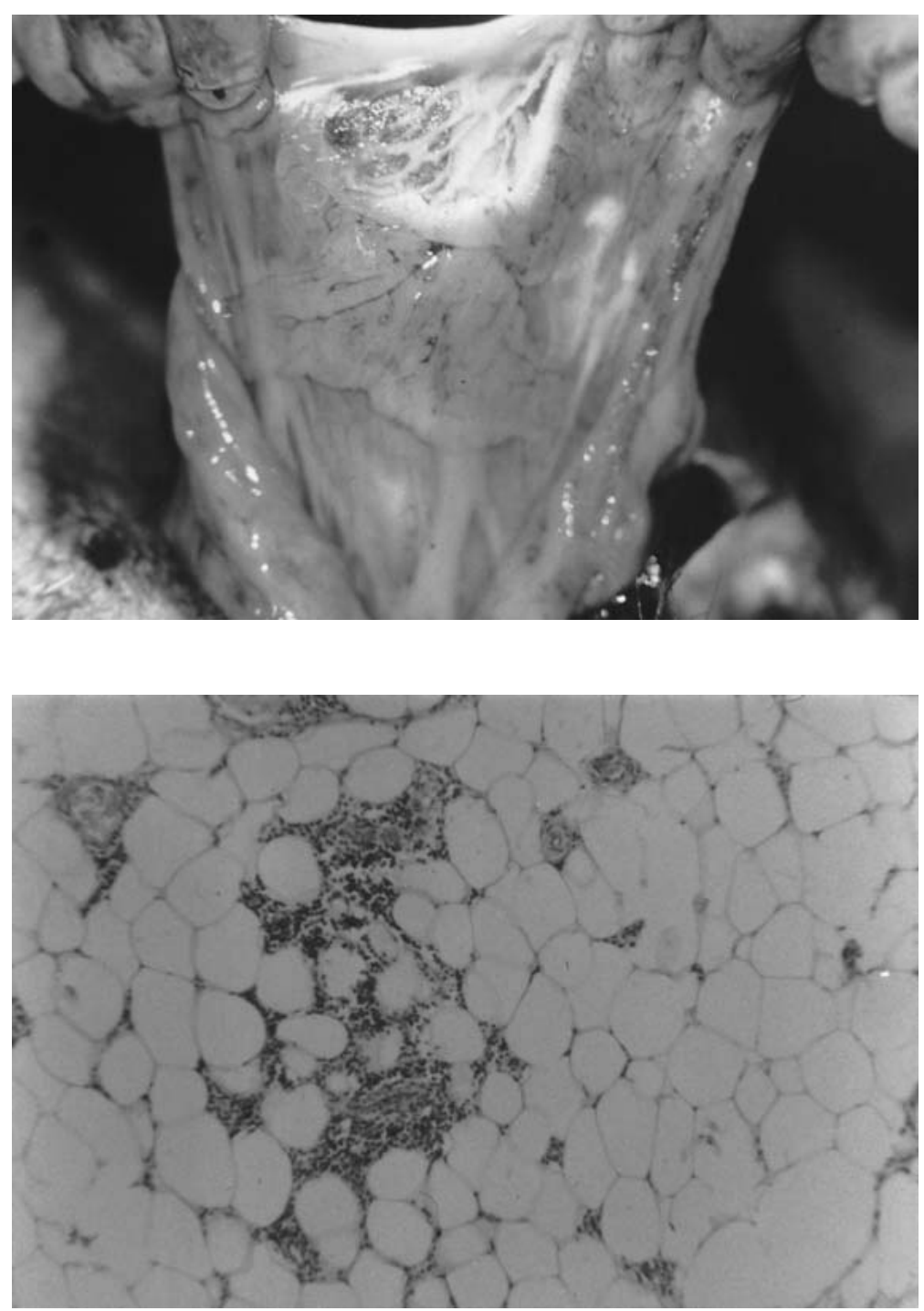

Fig. 2. Autopsy finding: 50 days after liposonication, the omentum has lost its fan appearance and the connective framework looks thickened and pale white.
Fig. 3. Histology of the omentum 50 days after liposonication: an aspecific chronic inflammatory reaction is present, consisting in small cells infiltrating the septa among the adipocytes and around the vessels (EE 200x). decreasing to $30-40$ pulses per minute until heart failure. Any attempt of resuscitation failed.

The two surviving animals were in good general conditions in the postoperative followup.

During post-mortem examination 50 days after the operation (autoptic phase), the whole omentum of the two surviving animals appeared twisted on itself, looking like a compact round mass (Fig. 2) and the connective network was thickened, presenting several fibrotic adherences to spleen and liver.

Evaluation of the histologic specimens (third phase) showed the development of an intense inflammatory reaction (Fig. 3), consisting of a cellular infiltrate with granulomatous patterns both inside the connective network and under the mesothelial lining and around the vessels. Fibrohyaline deposits were present under the mesothelium. On the contrary before sonication no hystopathological anomalies could be detected in the omentum.

The microscopic appearance of the parenchima of liver and spleen was normal.

\section{Discussion}

The dwarf-pig model was chosen for our experiment because of its abdominal cavity has similar anatomy to that of humans, and because of its functional capacity of storing fat in the omentum is analogous to humans.

The experiment was performed with standard anesthesiological and surgical techniques used in humans in order to be able to reproduce the procedures and to extrapolate data and suggestions obtained.

Some hypotheses were advanced about the high mortality rate obtained (50\% of the experimental animals).

It might be possible that the animals were not in fully good health, notwithstanding the basic preoperative laboratory checkup: actually they looked well-nourished, lively, and their preoperative electrocardiological tracks were absolutely normal.

It might be possible that ultrasounds, directed towards structures full of orto- and parasympathetic innervation, provoked lethal effects on the cardiac conductive system: these questions require further investigation on the side- 
effects of ultrasounds inside the abdominal cavity. Actually even low-temporal-average-intensity ultrasounds cause deleterious effects in lower organisms, through the mechanism of cavitation [1,2]. Besides, even weak ultrasounds are known to damage red blood cells [8].

We don't think that the high mortality rate can be due to the surgical learning curve, considering the high confidence of the operators both with experimental surgery and with ultrasound-assisted lipolysis.

Macro- and microscopic evaluations of the histologic specimens showed that an intense inflammatory reaction develops after surgery with fibrous alterations in the omentum and in the surrounding organs, even if the two pigs that had survived did not apparently suffer from any troubles.

Our results are discouraging and the technique seems to be ineffective and dangerous. However, further investigations are required in order to understand whether the inflammatory peritoneal reaction was specifically due to ultrasounds or only to the surgical handling of omentum.

\section{Conclusions}

Our preliminary data suggest that further experimental studies must be performed to delineate the real zone of injury and the total range of ultrasound effects on the omentum. Furthermore, it could be possible that ultrasounds are harmful for vital organs, particularly if transduced nearby their parenchima. This consideration must be taken into account also performing subcutaneous ul- trasound-assisted lipolysis, particularly when carried out on the trunk.

\section{References}

1. Carstensen EL: Acoustic cavitation and safety of diagnostic ultrasound. Ultrasound Med Biol 13:597-606, 1987

2. Daniels S, Kodama T, Price DJ: Damage to red blood cells induced by acoustic cavitation. Ultrasound Med Biol 21:105-111, 1995

3. Erol O, Spira M: Development and utilization of composite island flap employing omentum. Plast Reconstr Surg 65:415-418, 1980

4. Fournier PF: Liposculpture: the syringe technique. Blackwell, Paris, 1991

5. Fournier PF: Therapeutic megalipoextraction or megaliposculpture. Obesity Surgery 6:167, 1996

6. Fry WJ: Physical factors involved in ultrasonically induced changes in living systems: identification of non-thermal effects. Acoust Soc Am 22:867, 1950

7. Johnson SJ: An ultrasonic unit for treatment of Meniere's disease. Ultrasonics 5:173, 1967

8. Laborde JL, Hita A, Caltagirone JP, Gerard A: Fluid dynamics phenomena induced by power ultrasounds. Ultrasounds 38:297-300, 2000

9. Morrison R: Remarks on some functions of the omentum. Br Med J 1:76-78, 1906

10. Shaughnessy O: An experimental method of providing a collateral circulum to the heart. Br Surg 23:665, 1936

11. Zocchi M: New perspective in lipoplasty: the ultrasoundassisted lipectomy (UAL). In: Proceedings of the Congress of the French Society of Aesthetic Surgery, Paris, May 1988 